\title{
THE FIRST WHITEHEAD LEMMA FOR MALCEV ALGEBRAS ${ }^{1}$
}

\author{
RENATE CARLSSON
}

\begin{abstract}
In the present paper the first Whitehead lemma for separable Malcev algebras is proved, the dimensions being finite and the characteristic of the base field necessarily equal to zero. A consequence is the theorem of Malcev-Harish-Chandra for Malcev algebras. To get the lemma a structure theorem for modules over semisimple split Malcev algebras is proved.
\end{abstract}

1. Introduction. In this paper Whitehead's first lemma is proved for separable Malcev algebras. It says that every derivation of a separable Malcev algebra in a Malcev module is inner, the characteristic of the base field being zero. A consequence is the theorem of Malcev-Harish-Chandra for Malcev algebras.

These theorems are known for Lie algebras which are special Malcev algebras, for alternative, and for Jordan algebras. The lemma is proved by means of the classification of irreducible Malcev modules over simple split Malcev algebras. This classification is done in [1].

2. Definitions. Let $A$ be an algebra over a field $k$, the characteristic of $k$ not equal to $2 . A$ is a Malcev algebra if

(1) $x^{2}=0$

(2) $(x y \cdot z) t+(y z \cdot t) x+(z t \cdot x) y+(t x \cdot y) z=t y \cdot x z$ is satisfied for all $x, y, z, t \in A$.

If $R: A \rightarrow \operatorname{End}(A)$ is the (right) regular representation of $A$ with $R(x): y \mapsto y x$, then by (2) we have equivalently

$$
R(x y \cdot z)=R(x) R(y) R(z)-R(z) R(x) R(y)-R(y z) R(x)+R(y) R(z x) .
$$

A Malcev (bi-)module over a Malcev algebra $A$ over $k$ is a $k$-module $M$ with two bilinear compositions $A \times M \rightarrow M$ and $M \times A \rightarrow M$ so that $x m=-m x$ and (2) is valid for all $x, y, z \in A$ and $t \in M$. The semidirect sum $B$ $=A+M$ of $A$ and a Malcev module $M$ is a Malcev algebra, $B$ being the direct sum of the vectorspaces $A$ and $M$, with the multiplication $(x+m)$ $\cdot\left(x^{\prime}+m^{\prime}\right):=x x^{\prime}+x m^{\prime}+m x^{\prime}$ if $x, x^{\prime} \in A, m, m^{\prime} \in M$. $R$ denotes the regular representation of $B$ with $R(u): v \mapsto v u$ for $u, v \in B$.

A bimodule $M$ over $A$ is a Lie module if $x m=-m x$ and $m \cdot x y=m x \cdot y$

Received by the editors August 14, 1974.

AMS (MOS) subject classifications (1970). Primary 17A30, 17E05; Secondary 17B05, 17B15, $17 \mathrm{D} 05$.

Key words and phrases. Malcev algebras, Malcev modules, derivations, Whitehead's first lemma, theorem of Malcev-Harish-Chandra, Lie algebras.

${ }^{1}$ This paper is part of a dissertation submitted to the University of Hamburg, prepared under Professor Hel Braun. 
$-m y \cdot x$ for $m \in M$ and $x, y \in A$. Set $J(u, v, w):=u v \cdot w+v w \cdot u+w u$ $\cdot v, u, v, w \in B ; B$ as afore. The maximal Lie submodule $N_{M}:=\{m$ $\in M \mid J(m, x, y)=0$ for all $x, y \in A\}$ of a Malcev module $M$ over $A$ is the module nucleus. A derivation $D$ of $A$ in $M$ is a $k$-linear mapping $D: A \rightarrow M$ with $(x y) D=(x) D y+x(y) D$ if $x, y \in A$. $D$ is an inner derivation if $D$ can be extended to an inner derivation of $B$. The $k$-module of the inner derivations of $A$ in $M$ is generated by $\partial(x, m)$ and $r\left(m^{\prime}\right), x \in A, m \in M$, and $m^{\prime} \in N_{M}$, which are the restrictions from $B$ onto $A$ of the inner derivations $D(x, m)$ $:=(R(x), R(m))+R(x m)$ and $R\left(m^{\prime}\right)$ of $B,($,$) being the usual commutator$ product. Let $\rho$ be the canonic representation of $A$ in $M$ with $\rho(x): M \rightarrow M, m$ $\mapsto m x, x \in A$. In the subsequent, $A$ denotes a Malcev algebra, $M$ a Malcev module over $A$, both taken finite dimensional if nothing different is stated.

Let $\varphi: A \rightarrow k[x], k[x]$ the ring of the polynomials in $x$ over $k . \varphi$ is called a primary function if $\varphi(a)$ is irreducible for every $a \in A$, and $M_{\varphi}:=\{m$ $\left.\in M \mid(m)(\varphi(a)(\rho(a)))^{r}=0, r=r(a) \in \mathbf{N}, a \in A\right\}$ is the primary component of $M$ relative to $\varphi, \varphi$ is essential over $M$ if $M_{\varphi} \neq\{0\}$, and equally for $M_{\varphi}$. $\mathrm{d}(p)$ denotes the degree of $p \in k[x]$. If $\mathrm{d}(\varphi(a))=1$ for all $a \in A$ we have $M_{\alpha}=M_{\varphi}, M_{\alpha}$ the rootspace corresponding to the root $\alpha: A \rightarrow k, a \mapsto \alpha(a)$ with $\varphi(a)=x-\alpha(a)$. For nilpotent $A, M$ has a primary decomposition over $A$ into primary components, $M=\bigoplus M_{\pi_{i}}, i=1, \ldots, n, n \in \mathbf{N}[3] .\left\{\pi_{i}(a) \mid M_{\pi_{i}}\right.$ $\neq\{0\}, i=1, \ldots, n\}$ is the set of the irreducible factors of the minimum polynomial of $\rho(a) . A$ is splitting over $M$ if the characteristic roots of each $\rho(a)$ are in $k$. Then the primary decomposition is a rootspace decomposition.

Lemma 1. Let $A$ and $M$ be as above and $H$ a nilpotent subalgebra, splitting over $A$ and $M$. One has

(4) $A_{\alpha} A_{\beta} \subset A_{\alpha+\beta}$ for $\alpha \neq \beta, A_{\alpha}^{2} \subset A_{2 \alpha}+A_{-\alpha}$,

(5) $J\left(A_{\alpha}, A_{\beta}, A\right)=\{0\}$ if $\alpha \neq \beta \neq \gamma \neq \alpha$.

If $M$ is irreducible and non-Lie over $A$, we have

(6) $M_{\alpha} A_{\alpha} \subset M_{-\alpha}$ and $M_{\alpha} A_{\beta}=\{0\}$ if $\alpha \neq 0$ and $\beta \neq 0, \alpha,-\alpha$,

(7) $M_{\alpha} \neq\{0\} \Rightarrow A_{\alpha} \neq\{0\}$ or $A_{-\alpha} \neq\{0\}$.

If the characteristic of $k$ is 0 and $H$ is an abelian Cartan subalgebra, then

(8) $M_{0} H=\{0\}$ [1], [3].

3. Theorems. $A$ is split if $A$ contains a splitting Cartan subalgebra. The seven-dimensional simple split non-Lie Malcev algebra is denoted algebra of type $C_{M}^{-}$, and the regular module over $A$ module of type $C_{M}^{-}$. The twodimensional irreducible non-Lie Malcev module over the three-dimensional Lie algebra of type $A_{1}$ is called module of type $M_{2}$.

THEOREM 1. Let $A$ be a semisimple split Malcev algebra over a field of characteristic $0, M$ a Malcev module over $A, A=\bigoplus A_{i}, i=1, \ldots, n, n \in \mathbf{N}$, the decomposition into the direct sum of simple algebras. Then we have $M$ $=\bigoplus M_{r}, r=1, \ldots, m, m \in \mathbf{N}$, with the following properties: $M_{1}$ is the maximal Lie submodule of $M$. For each $r>1$ there exists $i, 1 \leqq i \leqq n$, so that $M_{r}$ is non-Lie and of type $M_{2}$ or $C_{M}^{-}$over $A_{i}$ and $M_{r} A_{j}=\{0\}$ if $j \neq i$ with $1 \leqq j \leqq n$. If $M$ is of type $M_{2}, A_{i}$ is a Lie algebra of type $A_{1}$, and in the second case $A_{i}$ is of type $C_{M}^{-}$.

Proof. Since $M$ is completely reducible over $A$ [3, Corollary 8], we can 
assume that $M$ is irreducible and not Lie. For semisimple $A$ the Cartan subalgebras are the self-normalizing nilpotent subalgebras [3].

Let $H$ be a splitting Cartan subalgebra of the split algebra $A$. Then $H_{i}:=H \cap A_{i}$ is a splitting Cartan subalgebra. of $A_{i}, i=1, \ldots, n$, and $H=\oplus H_{i}$. Let $\gamma_{i}$ be a root of $H_{i}$ in $A_{i}$, and $\gamma_{i}^{*}: H \rightarrow k$ the linear form defined by

$$
\gamma_{i}^{*}:= \begin{cases}\gamma_{i}(h) & \text { if } h \in H_{i} \\ 0 & \text { if } h \in H_{j}, j \neq i\end{cases}
$$

Then the essential roots of $H$ in $A$ are the extensions $\gamma_{i}^{*}$ of the essential roots $\gamma_{i}$ of each $H_{i}$ in $A_{i}$. One has $A_{\beta}=\left(A_{i}\right)_{\gamma_{i}}$ if $\beta=\gamma_{i}^{*} \neq 0$.

Let $\Delta$ denote the set of the essential roots of $H$ in $A, \Delta_{i}$ the set of the essential roots of $H_{i}$ in $A_{i}$ so that $\Delta=\cup \Delta_{i}^{*}, \Delta_{i}^{*}:=\left\{\gamma^{*} \mid \gamma \in \Delta_{i}\right\}, i$ $=1, \ldots, n$. If $H$ is splitting over $M$, we get from (7) that $M=\bigoplus M_{\beta}, \beta \in \Delta$, noting that $\beta \in \Delta$ implies $-\beta \in \Delta$. M is not a zero module, which means $A M \neq\{0\}$. Thus we get by (4) and (8) that there exists a weight $\gamma^{*} \in \Delta$, $\gamma^{*} \neq 0$, and $\gamma^{*} \in \Delta_{i}^{*}$ for a particular $i$. Set

$$
M^{\prime}:=\sum\left(M_{\beta^{*}}+M_{\beta^{*}} A_{-\beta^{*}}\right), \quad \beta \in \Delta_{i}, \beta \neq 0, \quad M^{\prime}=M^{\prime}(i) .
$$

We have $M^{\prime} \neq\{0\}$, and $M^{\prime} A_{i} \subset M^{\prime}$ by (4) and (6). For $j \neq i$ we get

$$
M^{\prime} A_{j}=\sum\left(M_{\beta^{*}} A_{j}+M_{\beta^{*}} A_{-\beta^{*}} \cdot A_{j}\right), \quad \beta \in \Delta_{i}, \beta \neq 0 .
$$

Now

$$
M_{\beta^{*}} A_{j}=M_{\beta^{*}} H_{j}+M_{\beta^{*}}\left(A_{j}\right)_{1},
$$

$\left(A_{j}\right)_{1}:=\bigoplus A_{\gamma^{*}}, \delta \in \Delta_{j}, \delta \neq 0$ (the Fitting one component of $A_{j}$ relative to $H_{j}$ ). Thus we get $M_{\beta^{*}} A_{j}=\{0\}$, for the first term in the sum is the zero space by (8) since $\beta^{*}(h)=0$ if $h \in H_{j}$, and the second is zero by (6). On the other hand we get

$$
J\left(M_{\beta^{*}}, A_{-\beta^{*}}, A_{j}\right)=J\left(M_{\beta^{*}}, A_{-\beta^{*}}, H_{j}\right)+J\left(M_{\beta^{*}}, A_{-\beta^{*}},\left(A_{j}\right)_{1}\right)=\{0\} \quad \text { by }(5) .
$$

Therefore

$$
M_{\beta^{*}} A_{-\beta^{*}} \cdot A_{j} \subset M_{\beta^{*}} A_{j} \cdot A_{-\beta^{*}}+M_{\beta^{*}} \cdot A_{-\beta^{*}} A_{j}=\{0\},
$$

since $M_{\beta^{*}} A_{j}=\{0\}$ and $A_{i} A_{j}=\{0\}$. Thus $M^{\prime}$ is a nonzero submodule of $M$, hence $M^{\prime}=M$, and we have $M A_{j}=\{0\}$ if $j \neq i$. $M$ is an irreducible non-Lie Malcev module over the simple Malcev algebra $A_{i}$. There exist exactly two cases [1]: Either $A_{i}$ is the three-dimensional Lie algebra of type $A_{1}$, and $M$ is a module of type $M_{2}$ over $A_{i}$, or $A_{i}$ is of type $C_{M}^{-}$, and $M$ is the regular module.

It remains to show that if $H$ is splitting over $A$, then it is splitting over $M$. Assume $H$ is not splitting over $M$. Let $M=\bigoplus M_{\pi_{i}}, i=1, \ldots, m$, be the decomposition of $M$ into essential primary components. Let $\bar{A}, \bar{M}, \bar{H}$ be the base field extensions of $A, M, H$ corresponding to the base field extension of $k$ to an algebraic closure $\bar{k} . \bar{H}$ is a Cartan subalgebra of the Malcev algebra $\bar{A}$ [3], 
splitting over $\bar{A}$ and $\bar{M}$. $\overline{A_{i}}$ is of the same type as $A_{i}$. For if $A_{i}$ is a Lie algebra, its Dynkin diagram is invariant by a base field extension. if $A_{i}$ is of type $C_{M}^{-}$, a standard basis of $A_{i}$ is a standard basis of $\overline{A_{i}}$, thus the type remains unchanged [5].

$\bar{M}$ is completely reducible over $\bar{A}$. We have $\left(\overline{A_{\beta}}\right)=(\bar{A})_{\bar{\beta}}, \beta \in \Delta, \bar{\beta}$ the linear extension of $\beta$ to $\bar{H}$, by the linearity of the essential roots if char $k=0$. We get $\left(\overline{M_{\pi_{i}}}\right)=\bigoplus\left(\overline{M_{\pi_{i}}}\right)_{\gamma_{i j}}, j=1, \ldots, m=m(i) \in \mathbf{N}, \gamma_{i j}: \bar{H} \rightarrow \bar{k}$ an essential root of $H$ over $\bar{M}$. Every rootspace $M_{\beta}$ generates a submodule $M^{\prime}$ over $A$ with $H$ splitting over $M^{\prime}$. This is obvious by (4), considering the canonic imbedding in $\bar{M}$. Thus for any $i$ we have $\max \left\{\mathrm{d}\left(\pi_{i}(h)\right) \mid h \in H\right\} \geqslant 2$. By (7) there exists an essential root $\gamma=\gamma_{i j}$ of $\bar{H}$ over $\bar{M}, \gamma=\bar{\beta}, \beta \in \Delta_{i}^{*}$. For this $i$ and any $h \in H$ we have $x-\gamma(h) \mid \pi_{i}(h)$. From $\gamma(h)=\beta(h) \in k$ and the irreducibility of $\pi_{i}(h)$ we obtain $\mathrm{d}\left(\pi_{i}(h)\right)=1$, which is a contradiction. $H$ is splitting over $M$.

A Malcev algebra is separable if every base field extension of the algebra is semisimple. We now get

THeORem 2. (The first Whitehead lemma for Malcev algebras.) Let $A$ be a separable Malcev algebra over a field of characteristic 0 , and $M$ a Malcev module over $A$. Let $D$ be a derivation of $A$ in $M$. Then $D$ is an inner derivation.

Proof. Let $A$ be split and semisimple. Since $M$ is completely reducible over $A$ we can restrict ourselves to the case of irreducible $M$. Let $M$ be a zero module, $M A=\{0\}$. It is well known and trivial that $D$ is inner, for $(A) D=\left(A^{2}\right) D \subset(A) D A \subset M A=\{0\}$ so that $D=r(0)$.

If $A$ is simple and non-Lie, $A$ is of type $C_{M}^{-}$. Either $M$ is the zero module or regular [1]. Since every derivation of $A$ is inner [5, p. 455], $D$ is an inner derivation.

Let $A$ be semisimple and $M$ an $A$ Lie module. If $A$ is a Lie algebra then it is well known that all Lie derivations are inner. Otherwise we have $A=C E$ where $C$ is a Lie algebra and $E$ a direct sum of algebras of type $C_{M}^{-} . M$ is a Lie module over $E$, so we have by [1] that $M E=\{0\}$ and, therefore, $(E) D=\{0\}$. The restrictions of $D$ onto $C$ and $E$ are derivations of $C$ and $E$ into $M$. So there exists $m \in M$ with $(x) D=x m$ for all $x \in C$. If $y \in E$ then one has $y m=0$; thus $D=\mathbf{r}(m)$.

Let $M$ be non-Lie. From Theorem 1 we get that there exists an $i$ so that either $A_{i}$ is of type $C_{M}^{-}$, and $M$ regular over $A_{i}$, and thus the restriction of $D$ onto $A_{i}$ is inner, or $A_{i}$ is of type $A_{1}$ and $M$ of type $M_{2}$. Then the restriction of $D$ onto $A_{i}$ is inner by [1]. Thus we have $D=D^{\prime}:=\sum \partial\left(x_{r}, m_{r}\right)$ on $A_{i}$, $r=1, \ldots, s, s \in \mathbf{N}$ with $x_{r} \in A_{i}$ and $m_{r} \in M$. Since $M A_{j}=\{0\}$ if $j \neq i$, it follows that $D=D^{\prime}$.

Let $A$ be separable, and $\tilde{k}$ be an extension of $k$ so that the corresponding base field extension $\tilde{A}$ of $A$ is split. Let $\tilde{M}$ denote the base field extension of $M$. $D$ defines an inner derivation $\tilde{D}$ of $\tilde{A}$ in $\tilde{M}$ which extends $D$. As is known, it follows that already $D$ is inner.

Corollary 1. Let $A$ and $M$ be as in the theorem, $D: A \rightarrow M$ a derivation. Then

$$
D=\sum \partial\left(x_{i}, m_{i}\right), \quad i=1, \ldots, r, r \in \mathbf{N}, x_{i} \in A, m_{i} \in M .
$$


Proof. Because $N_{M}$ is completely reducible over $A$, we can assume for a derivation $r(m), m=\sum x_{i} m_{i}, x_{i} \in A_{i}, m_{i} \in N_{M}, i=1, \ldots, s, s \in \mathbf{N}$. For $m \in N_{M}$ and all $x, y \in A$ we get

$$
(x) r(y m)=(x) R(y m)=(x)(R(y), R(m))=(x) \partial\left(\frac{1}{2} y, m\right) .
$$

COROLlaRy 2. Let $A$ be a Malcev algebra and $C$ a separable subalgebra of $A$, the characteristic of $k$ being 0 . Then every derivation of $C$ in $A$ can be extended to an inner derivation of $A$.

For two ideals $I$ and $B$ of $A$ define the ideal operator $K_{B}$ by

$$
K_{B}(I):=I B+I B \cdot A \cdot K_{B}(I)
$$

is an ideal of $A$. Set $K_{B}^{0}(I):=I, K_{B}^{n}:=K_{B} K_{B}^{n-1}, n \in \mathbf{N}$. If there exists $n \in \mathbf{N}$ with $K_{I}^{n}(I)=\{0\}, I$ is $K$-nilpotent. We have (no condition on the dimension)

Lemma 2. Let $I$ be an ideal of the Malcev algebra $A$, char $k \neq 2$. I is nilpotent exactly if $I$ is $K$-nilpotent.

Proof. $\quad$ 1. $I^{2 n} \subset K_{I}^{n}(I)$. For by (3) each product of $2 n$ elements of $I$ is a linear combination of right normal products in $r \geqq n+1$ elements out of $I$.

2. $K_{I}^{2 n}(I) \subset I^{n+1}$ follows by induction on $n$. Assuming it for $n \geqq 0$ we get by (2),

$$
K_{I}^{2(n+1)}(I)=K_{I}^{2}\left(K_{I}^{2 n}(I)\right) \subset K_{I}^{2 n}(I) I \subset I^{n+2} .
$$

If $A$ is a Malcev algebra, $A=B \oplus S, B$ a semisimple subalgebra, $S$ the solvable radical of $A$, then the decomposition is a Wedderburn or Levi decomposition and $B$ a Wedderburn or Levi factor of $A$. If $U$ and $V$ are subalgebras, $f$ an automorphism of the algebra, and $(U) f=V, U$ and $V$ are conjugate. An inner automorphism of $A$ is a product of automorphisms $\exp (D)$ where $D$ is a nilpotent inner derivation of $A$.

We then get further:

THEOREM 3. (Theorem of Malcev-Harish-Chandra for Malcev algebras.) Let $A$ be a Malcev algebra over a field of characteristic 0 , and $A=B \oplus S a$ Wedderburn decomposition. If $C$ is a separable subalgebra then there exists an inner automorphism $f$ of the algebra $A$ so that $(C) f \subset B$.

The proof follows by modifying Harish-Chandra's proof (cf. [2]) for Lie algebras. To this aim one has to substitute the ordinary solvability and nilpotency by $L$-solvability and $K$-nilpotency which reduce the defining conditions to conditions on ideals, and to observe Corollary 1.

We have again:

COROllary 3. If $A$ has a Wedderburn decomposition then every separable subalgebra is a subalgebra of a Wedderburn factor.

Corollary 4. Let $S$ be the radical of $A, A / S$ separable. Then any two Wedderburn factors are conjugate by an inner automorphism.

\section{REFERENCES}

1. R. Carlsson, Malcev-Moduln, J. Reine Angew. Math. (to appear). 
2. N. Jacobson, Lie algebras, Interscience Tracts in Pure and Appl. Math., no. 10, Interscience, New York and London, 1962. MR 26 \# 1345.

3. E. N. Kuz'min, Mal'cev algebras and their representations, Algebra i Logika 7 (1968), no. 4, 48-69 = Algebra and Logic 7 (1968), 233-244. MR 40 \#5688.

4. - The locally nilpotent radical of Mal'cev algebras satisfying the nth Engel condition, Dokl. Akad. Nauk SSSR 177 (1967), 508-510 = Soviet Math. Dokl. 8 (1967), 1434-1436. MR 36 \#2660.

5. A. A. Sagle, Malcev algebras, Trans. Amer. Math. Soc. 101 (1961), 426-458. MR 26 \# 1343.

6. E. J. Taft, The Whitehead first lemma for alternative algebras, Proc. Amer. Math. Soc. 8 (1957), 950-956. MR 19, 728.

Mathematisches Seminar, Universität Hamburg, 2 Hamburg 13, Germany 